\title{
Imaging defects and their evolution in a metal- organic framework at sub-unit-cell resolution
}

\author{
Lingmei Liu' ${ }^{1,9}$, Zhijie Chen $\mathbb{1}^{2,9}$, Jianjian Wang ${ }^{1,3,9}$, Daliang Zhang ${ }^{4 \star}{ }^{4}$, Yihan Zhu ${ }^{5}$, Sanliang Ling ${ }^{6}$, \\ Kuo-Wei Huang $\mathbb{D}^{7}$, Youssef Belmabkhout $\mathbb{1}^{2}$, Karim Adil $\mathbb{D}^{2}$, Yuxin Zhang ${ }^{3}$, Ben Slater ${ }^{8 *}$, \\ Mohamed Eddaoudi ${ }^{2 \star}$ and Yu Han ${ }^{1,7 \star}$
}

Defect engineering of metal-organic frameworks (MOFs) offers promising opportunities for tailoring their properties to specific functions and applications. However, determining the structures of defects in MOFs-either point defects or extended ones-has proved challenging owing to the difficulty of directly probing local structures in these typically fragile crystals. Here we report the real-space observation, with sub-unit-cell resolution, of structural defects in the catalytic MOF UiO-66 using a combination of low-dose transmission electron microscopy and electron crystallography. Ordered 'missing linker' and 'missing cluster' defects were found to coexist. The missing-linker defects, reconstructed three-dimensionally with high precision, were attributed to terminating formate groups. The crystallization of the MOF was found to undergo an Ostwald ripening process, during which the defects also evolve: on prolonged crystallization, only the missing-linker defects remained. These observations were rationalized through density functional theory calculations. Finally, the missing-cluster defects were shown to be more catalytically active than their missing-linker counterparts for the isomerization of glucose to fructose.

M etal-organic frameworks (MOFs) are crystalline porous materials consisting of metal clusters (nodes) and organic coordinating ligands (linkers) ${ }^{1-8}$. In addition to manipulating the periodic bulk structure, defect tuning in MOFs has attracted considerable research attention because it offers a means to locally tune porosity, create open-metal sites and modulate surface properties, and thus has important implications for separation, storage, catalysis, charge transport and mechanical responses ${ }^{9-15}$. UiO-66 is one of the most widely studied MOFs, largely due to its exceptional chemical stability ${ }^{16}$ and catalytic activity, which is linked to the presence of structural defects ${ }^{15}$. UiO-66 consists of hexanuclear clusters, six-centred metal ( $\mathrm{Zr}$, Hf or U) oxyhydroxides, each connected to 12 benzene-1,4-dicarboxylate (BDC) linkers (12-connected nodes; fcu net) with the nominal chemical formula $\left[\mathrm{M}_{6} \mathrm{O}_{4}(\mathrm{OH})_{4}\right]\left[\mathrm{C}_{6} \mathrm{H}_{4}(\mathrm{COO})_{2}\right]_{6}(\text { Fig. 1a })^{16-18}$. Missing-linker and missing-cluster defects, which respectively refer to the omission of a number of linkers and nodes from the perfect crystallographic structure, have been inferred to exist in UiO-66, but the exact structures of these defects and their spatial distribution remain ambiguous ${ }^{19-24}$.

Non-stoichiometric compositions and unusual gas sorption behaviours are often used to affirm the presence of defects in UiO$66^{19-21}$. However, these proxies do not provide the atomic detail of the defect structures. Neutron powder diffraction ${ }^{22}$ and synchrotron single-crystal X-ray diffraction ${ }^{23,24}$ showed that a seemingly perfect UiO-66( $\mathrm{Zr})$ contains $\sim 10 \%$ of missing linkers. A combination of X-ray diffraction, electron diffraction and anomalous X-ray scattering identified the ordering of missing-cluster defects into nanoregions in a UiO-66(Hf) sample ${ }^{25}$. Fluorescence lifetime imaging revealed the spatial distribution of defects within individual UiO-67 (an isoreticular MOF of UiO-66) crystals at submicrometre resolution $^{26}$. It is also known that the concentration of defects in UiO-66 can be tuned by adding various terminal monocarboxylic acids in the synthetic system as modulators ${ }^{19}$. However, despite numerous investigations of structural defects in UiO-66, many questions remain. What are the exact structures of the defects? Do the missing-linker defects form locally ordered domains as has been suggested for the missing-cluster defects? ${ }^{25}$ Are there missing-linker defects and missing-cluster defects coexisting in the same UiO-66 crystal? Can defect transformation occur during crystallization? All of these questions cannot be answered without a technique that permits such defects to be probed directly.

High-resolution transmission electron microscopy (HRTEM) is a powerful tool to directly observe individual defects in real space, at atomic resolution ${ }^{27}$. However, conventional HRTEM is unsuitable for studying MOFs because the electron beam can easily damage their structures. High-angle annular dark-field scanning TEM has been used to observe metal clusters ${ }^{28-31}$ and lattice distortion ${ }^{28}$ in MOFs, but it did not disclose more structural details due to the limited resolution and weak contrast of organic components. Here, we have combined the recently developed low-dose HRTEM technique $^{32,33}$ with electron crystallography to investigate defects in MOFs by real-space direct imaging. These observations provide insight into the defects in UiO-66, including their types, distributions and correlations, their exact three-dimensional (3D) structures, as well as their evolution.

'King Abdullah University of Science and Technology (KAUST), Physical Sciences and Engineering Division, Advanced Membranes and Porous Materials (AMPM) Center, Thuwal, Saudi Arabia. ${ }^{2}$ KAUST, Physical Sciences and Engineering Division, AMPM Center, Functional Materials Design, Discovery and Development Research Group (FMD3), Thuwal, Saudi Arabia. ${ }^{3}$ Multi-scale Porous Materials Center, Institute of Advanced Interdisciplinary Studies, Chongqing University, Chongqing, China. ${ }^{4}$ KAUST, Core Labs, Thuwal, Saudi Arabia. ${ }^{5}$ Department of Chemical Engineering, Zhejiang University of Technology, Hangzhou, China. ${ }^{6}$ Advanced Materials Research Group, Faculty of Engineering, University of Nottingham, University Park, Nottingham, UK. ${ }^{7}$ KAUST, KAUST Catalysis Center (KCC), Thuwal, Saudi Arabia. ${ }^{8}$ Department of Chemistry, University College London, London, UK. ${ }^{9}$ These authors contributed equally: Lingmei Liu, Zhijie Chen, Jianjian Wang. *e-mail: daliang.zhang@kaust.edu.sa; b.slater@ucl.ac.uk; mohamed.eddaoudi@kaust.edu.sa; yu.han@kaust.edu.sa 
a

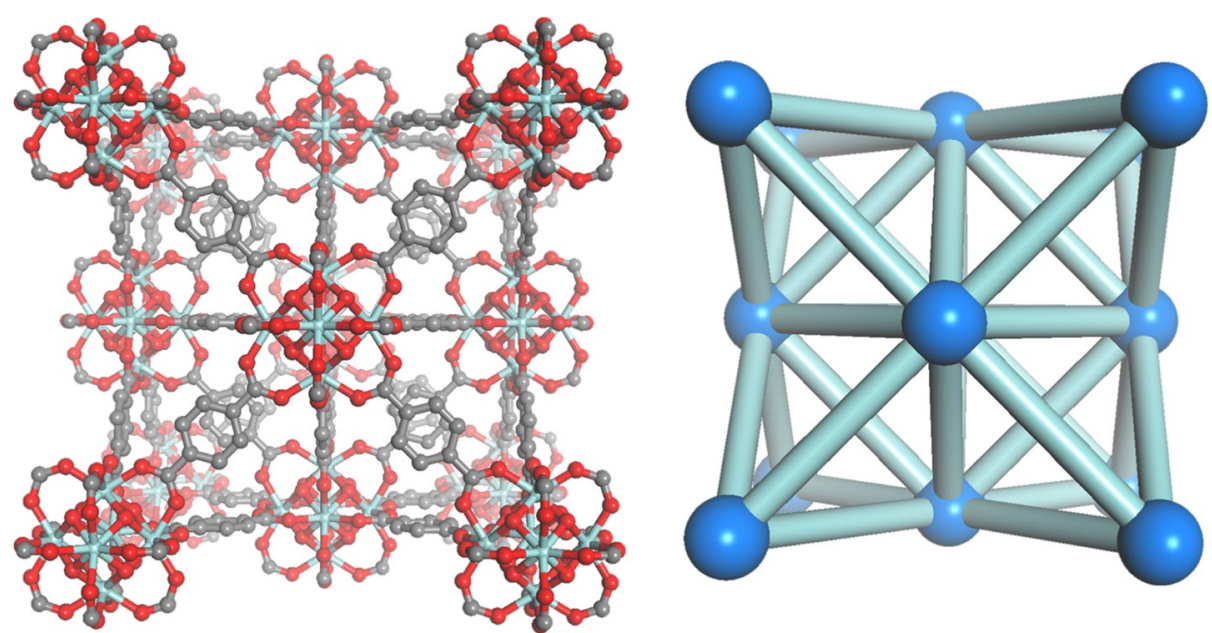

b

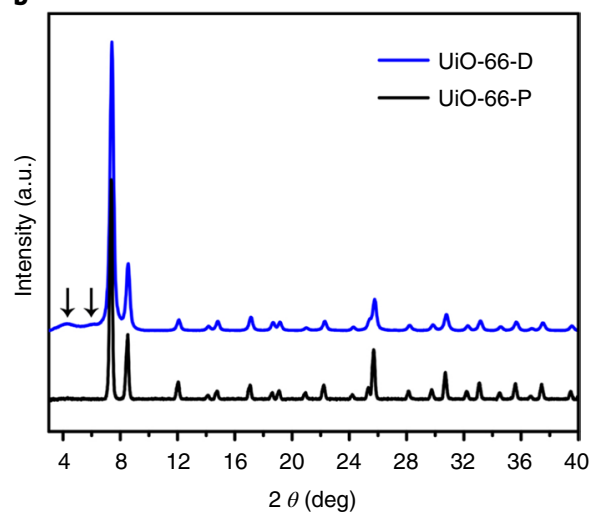

c

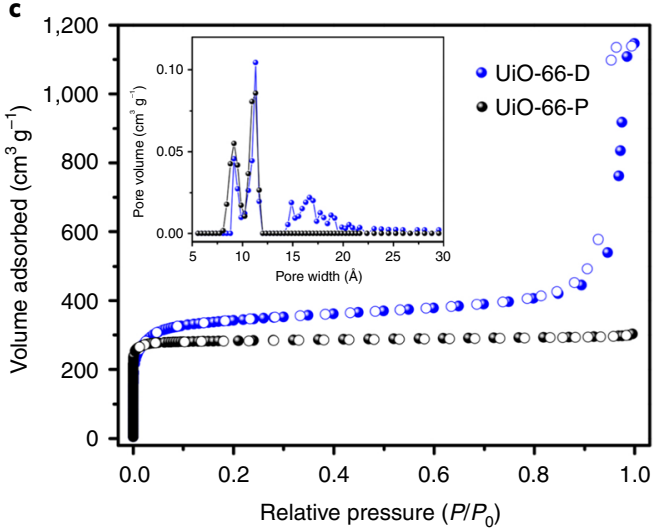

Fig. 1 | Structural illustration and characterizations of UiO-66 samples. a, Left, crystallographic model of the perfect UiO-66 structure ( $\mathrm{Zr}$, cyan; O, red; $\mathrm{C}$, grey; $\mathrm{H}$ atoms are omitted for clarity). Right, corresponding topological representative; the blue spheres represent the metal clusters, and the teal rods represent the ligands. b, Powder X-ray diffraction patterns of UiO-66-P and UiO-66-D (the nearly defect-free, 'perfect' UiO-66 sample and the defective one, respectively). Black arrows indicate the broad diffuse scattering peaks. $\mathbf{c}, \mathrm{N}_{2}$ sorption isotherms and the derived pore size distribution profiles (inset) of the two materials. Compared to UiO-66-P, UiO-66-D has an extra pore size distribution centred at $\sim 1.7 \mathrm{~nm}$, which is not associated with the interparticle porosity resulting in the significant adsorption at the high relative pressure region $\left(P / P_{0}>0.9\right)$.

\section{Results and discussion}

Observation and 3D reconstruction of missing-linker defects. A defective UiO-66 sample (denoted UiO-66-D) was synthesized using formic acid (50 molar equivalents relative to BDC) as a modulator to promote the formation of defects. The powder X-ray diffraction (PXRD) pattern of UiO-66-D exhibits two broad, weak yet discernible peaks at a $2 \theta$ of $\sim 4^{\circ}$ and $\sim 6^{\circ}$ (Fig. 1b), which are considered indicative of the presence of ordered missing-cluster nanoregions ${ }^{25}$. Analysis of the $\mathrm{N}_{2}$ adsorption isotherm shows that UiO-66-D has a marked pore size distribution centred at $1.7 \mathrm{~nm}$, in addition to the primary pores associated with the crystallographic structure (Fig. 1c). For comparison purposes, a nearly defect-free UiO-66 sample (UiO-66-P) was prepared and characterized; this does not show diffuse scattering features in the PXRD or extra pores of $1.7 \mathrm{~nm}$ (Fig. 1). These comparative results validate the reliability of the measurements, confirming that UiO-66-D includes a larger number of structural defects.

The HRTEM images presented in Figs. 2 and 3 were acquired from different areas of UiO-66-D consisting of interconnected irregular small $(<50 \mathrm{~nm})$ particles. Their contrasts were processed to be more directly interpretable by correcting the effect of the contrast transfer function (CTF) of the objective lens. Raw HRTEM images and low-magnification TEM images are provided in Supplementary
Figs. 1 and 2, respectively. The images shown in Fig. 2a,b match the projected structural models and simulated potential maps of the perfect UiO-66(Zr) structure $(F m-3 m ; a=20.7 \AA)$ along the [001] and [110] directions, respectively. Interestingly, Fig. $2 \mathrm{~d}$ has the same projection direction as Fig. $2 \mathrm{~b}$, but shows different image contrasts with apparent features of a missing linker: the contrasts of the horizontally arranged BDC ligands observed in Fig. $2 \mathrm{~b}$ are not present in Fig. 2d (indicated by red arrows). As a consequence, adjacent opposing triangle-shaped channels in Fig. $2 \mathrm{~b}$ are vertically merged into rhombus-shaped channels in Fig. 2d. A similar phenomenon is observed for the [001] images: the contrasts of benzene rings in the $\mathrm{BDC}$ ligands that are encompassed by four $\mathrm{Zr}_{6} \mathrm{O}_{8}$ clusters are clearly observed in Fig. 2a but not in Fig. 2c. These results indicate that, besides 'perfect' regions (Fig. 2a,b), UiO-66-D has ordered regions consisting of missing-linker defects (Fig. 2c-e). In fact, the perfect structure is a minor component in $\mathrm{UiO}-66-\mathrm{D}$ while most areas are defective with missing-linker features, according to the HRTEM observations. The image in Fig. 2e shows a square arrangement of $\mathrm{Zr}_{6} \mathrm{O}_{8}$ clusters but a low plane symmetry of $p 2 \mathrm{~mm}$, suggesting that missing-linker defects give rise to a decrease in symmetry along one of the originally equivalent $<001>$ directions.

By combining the HRTEM images from three distinct projections (Fig. 2c-e), the unit cell of the missing-linker region is determined 

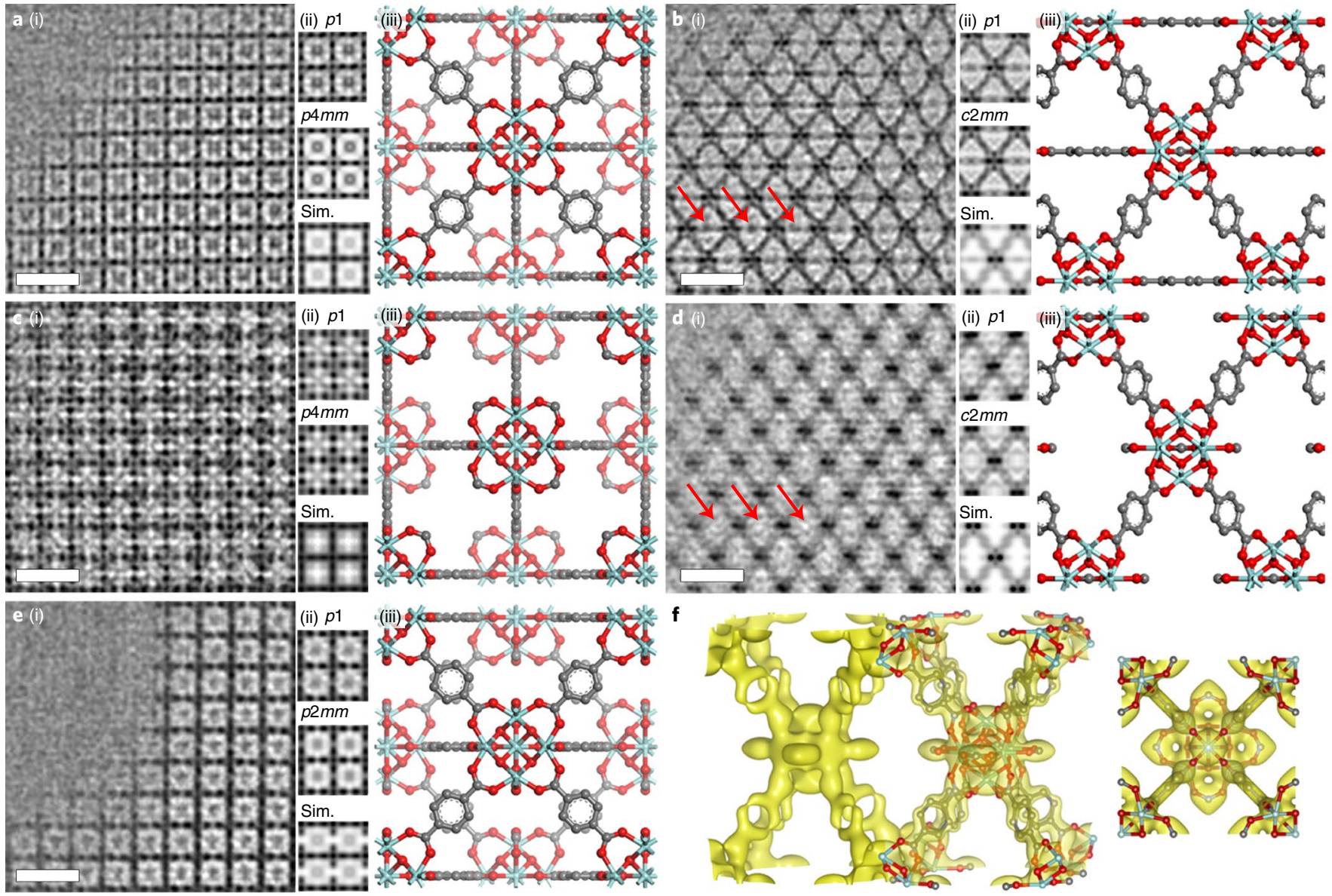

Fig. 2 | HRTEM analysis of perfect and missing-linker regions in UiO-66-D. a-e, CTF-corrected HRTEM images and structural models along the [001] (a) and [110] (b) zone axes of perfect UiO-66 (Fm-3m), and along the [001] (c), [100] (d) and [110] (e) zone axes of the missing linker defect (14/mmm). In each panel: (i) CTF-corrected image; (ii) p1-averaged image (top), symmetry-imposed image (middle) and simulated projected potential (bottom); (iii) the projected structural model. The $p 1$-averaged images are equivalent to projected electrostatic potential maps, which are calculated from the Fourier summation of reflection peaks in the Fourier transform of the original HRTEM images. The symmetry-imposed images are calculated in a similar way, except that symmetry-related reflections are averaged in amplitude before Fourier summation. The similarity between the $p 1$-averaged and symmetryimposed images validates the symmetry determination. The similarity between the processed HRTEM image and the simulated projected potential in c, $\mathbf{d}$ and $\mathbf{e}$ validates the structural model of the missing-linker defect. f, Reconstructed 3D electrostatic potential map viewed in two different orientations, with the structural model of the missing-linker defect superimposed, showing an 8-connected network with the $\mathrm{Zr}_{6} \mathrm{O}_{8}$ clusters, $\mathrm{BDC}$ linkers and defectterminating ligands (formates), which are all well resolved. In all structural models, the atom colour code is the same as described in Fig. 1, with $\mathrm{H}$ atoms omitted for clarity. Scale bars, $2 \mathrm{~nm}$.

as tetragonal with $a_{\mathrm{ML}}=a / \sqrt{ } 2$ and $c_{\mathrm{ML}}=a$, where $a$ is the unit cell parameter of the parent UiO-66 and $a_{\mathrm{ML}}$ and $c_{\mathrm{ML}}$ are the unit cell parameters of the missing-linker structure. The three images are thus indexed to the [001], [100] and [110] zone axes, respectively, according to the tetragonal crystal system. The observed reflection conditions $(h+k+l=2 n)$ from Fourier transforms of the HRTEM images indicate an $I$-centred lattice (Supplementary Fig. 3). Given that the [001], [100] and [110] images have projected symmetries of $p 4 \mathrm{~mm}, \mathrm{c} 2 \mathrm{~mm}$ and $p 2 \mathrm{~mm}$, respectively, two space groups, $I 4 / \mathrm{mmm}$ (no. 139) and $I 422$ (no. 97), are possible. We reconstructed the missing-linker defect using the electron crystallography method, which renders a 3D electrostatic potential map from the Fourier summation of the crystal structure factors determined from the HRTEM images $^{34,35}$. The reconstruction using space group of $I 4 / \mathrm{mmm}$ or I422 gave similar results (Supplementary Fig. 4). The reconstructed potential map clearly shows that the $\mathrm{Zr}_{6} \mathrm{O}_{8}$ clusters retain the same geometry as in the parent UiO-66; however, each cluster becomes 8-connected rather than 12-connected with neighbouring clusters, converting the unit cell from a face-centred cubic to a body-centred tetragonal structure (Fig. 2f). The resulting framework follows the underlying topology of a bcu net. The high precision of the reconstruction allows us to identify not only the $\mathrm{Zr}_{6} \mathrm{O}_{8}$ clusters and $\mathrm{BDC}$ ligands, but also the charge-compensating terminal formate ligands that substitute the missing bridging ligands to cap the open metal sites (Fig. $2 \mathrm{f}$ and Supplementary Fig. 5). The missing-linker defect can therefore be described as the result of the removal of all faceon BDC ligands from the parent UiO-66 structure when it is projected along one of the $<001\rangle$ zone axes (Fig. 2a,c). The simulated $2 \mathrm{D}$ projected potential maps of this structure perfectly match the HRTEM images, in all directions (Fig. $2 \mathrm{c}-\mathrm{e}$ ), which attests that the structure of the missing-linker defect has been correctly solved. We note that there is inconsistency in the literature with regard to defect termination in UiO-66: many papers argue for termination by monocarboxylates (that is, formates when using formic acids as modulators $)^{19,36,37}$, whereas one study concluded that the defective sites are capped by water molecules and charge-balanced by hydroxides ${ }^{24}$. We used liquid chromatography, ${ }^{13} \mathrm{C}$ NMR spectroscopy and mass spectrometry to analyse HF-dissolved or thermally decomposed UiO-66-D samples (Supplementary Fig. 6). The results provide substantive evidence that formates are present in this 

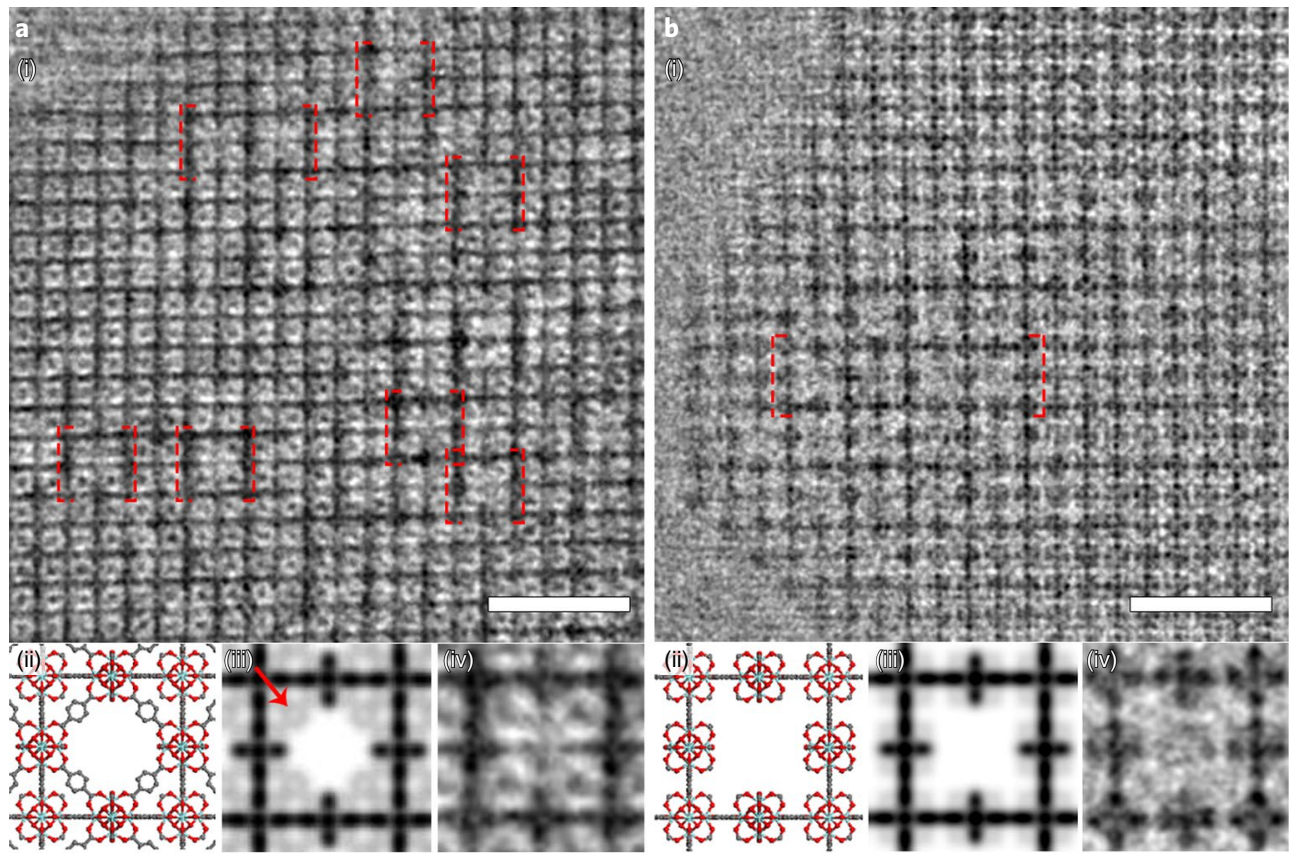

Fig. 3 | HRTEM analysis of the missing-cluster defects in UiO-66-D. a,b, CTF-corrected HRTEM images of the [001] direction, showing different types of missing-cluster defects, adopting the reo structure (a) and the scu structure (b). In each panel: (i) CTF-corrected HRTEM images (scale bars, $5 \mathrm{~nm}$ ); (ii) projected structural model (with the same atom colour code as in Fig. 1); (iii) the simulated projected potential map; (iv) the experimental image (processed by real-space averaging to enhance the signal-to-noise ratio) for comparison. The unit cells in panels (i), shown by red dashed boxes, are extracted as individual images, aligned with each other, and then merged to generate the real-space averaged image. The contrast associated with the $\mathrm{BDC}$ linkers surrounding the missing cluster is indicated by a red arrow in the simulated potential map in (a(iii)).

material $(\sim 4 \mathrm{wt} \%)$, corroborating the structural model we reconstructed from HRTEM.

Identification of two types of missing-cluster defect. The missinglinker defect has the same extinction as the parent UiO-66 structure (Supplementary Fig. 7), and therefore does not account for the observed diffuse scattering in the PXRD. The diffuse peaks in the PXRD have been correlated with short-range ordered missingcluster defects, but evidence from HRTEM imaging could not be obtained due to beam damage ${ }^{25}$. In this HRTEM study, we occasionally observed, in different incidences, weak superlattice reflections that correspond to the extinct 100 and 110 reflections of pristine $\mathrm{UiO}-66$, in agreement with the diffuse peaks in the PXRD. We also identified, in certain areas, nanosized domains with ordered cluster vacancies, from which the superlattice reflections originate (Supplementary Fig. 8). These observations directly correlate superlattice reflections with the missing-cluster defects. The observed missing-cluster domains are always only a few unit cells in dimension, much smaller than the missing-linker domains.

Figure 3 shows typical [001] images containing missing-cluster domains. We note that the image contrast around the missing cluster differs between different domains. Figure $3 a, b$ presents two scenarios, in which the face-on BDC linkers, which are surrounding but not connected to the missing cluster, are present and absent, respectively. The first scenario is in good agreement with the missing-cluster model proposed by Matthew Cliffe and co-workers ${ }^{25}$ that follows the underlying topology of an 8-connected reo net (Fig. 4). The second scenario, however, cannot be explained by the reo model, suggesting the coexistence of a different type of missing-cluster defect. The image contrast observed in Fig. $3 \mathrm{~b}$ allows us to directly deduce a structural model that follows the underlying topology of an scu net, having one-third of the $\mathrm{Zr}_{6} \mathrm{O}_{8}$ clusters 8-connected with surrounding clusters through BDC linkers, and the other two-thirds 4-connected (Fig. 4). This scu model is
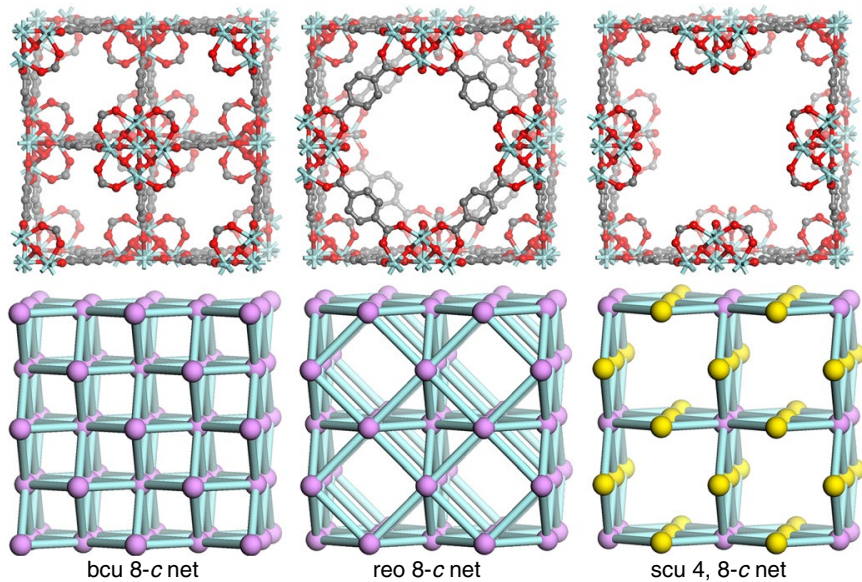

Fig. 4 | Illustrations of various defective structures in UiO-66. Top, crystallographic structural models (with the same atom colour code as in Fig. 1). Bottom, corresponding topological representatives $(2 \times 2 \times 2)$ of the 8-connected missing-linker defects (bcu net; transitivity [1112]), 8-connected missing-cluster defects (reo net; transitivity [1122]) and the 4,8-connected missing-cluster defects (scu net; transitivity [2133]). The transitivities [pqrs] denote $p$ types of vertices, $q$ types of edges, $r$ types of faces and $s$ types of tiles. Purple and yellow spheres indicate 8 - and 4-connected nodes, respectively.

validated by the HRTEM images of other directions containing missing-cluster defects (Supplementary Figs. 8 and 9). The reo structure is realized by removing 1 out of 4 symmetry-equivalent $\mathrm{Zr}_{6} \mathrm{O}_{8}$ clusters along with the associated BDC linkers from each unit cell of the ideal UiO-66, while the scu model can be considered as 
removing clusters in a similar way, but from the bcu missing-linker structure (Fig. 4). Therefore, the scu model has the same arrangement of clusters but it is more deficient in linkers compared to the reo model.

Because the scu structure has projections that are similar to those of the reo structure in some orientations (Supplementary Fig. 10), it is not always possible to distinguish them in HRTEM, although the missing clusters can be easily identified from the image contrast. Besides, the image contrast can be smeared and difficult to interpret if there are multiple structures and/or different orientations overlapping along the projection direction. In the present study, this issue was largely avoided by choosing ultrafine crystallites $(\sim 20 \mathrm{~nm})$ for imaging. In addition to well-defined reo and scu missing-cluster defects, we also observed large cavities in the UiO66-D crystals resulting from irregular, continuous cluster vacancies (Supplementary Fig. 11). These results indicate the inhomogeneity in the local structure of this material.

Relative stabilities of different defects. We performed detailed density functional theory (DFT) calculations to infer the formation energies of missing-linker and missing-cluster defects from a comparison of the bcu, reo and scu structures with respect to the perfect fcu structure. We find that the relative stability follows the order $\mathbf{f c u}>\mathbf{b c u}>\mathbf{r e o}>\mathbf{s c u}$ and that the absolute defect formation energies for missing-linker and missing-cluster defects are reasonably small, consistent with the fact that defects can be readily located by HRTEM, implying that they have a significant concentration (see additional discussions in Supplementary Table 1). The order of stability correlates with the connectivity in general. fcu is most stable due to its fully 12-connected network. The absolute defect formation energies of bcu and reo are similar, +17.8 and $+20.4 \mathrm{~kJ} \mathrm{~mol}^{-1}$ per three $\mathrm{Zr}^{4+}$, respectively, which is expected because their connectivity and stoichiometry are identical (8-connected net); reo is less stable because it contains missing clusters leading to a lower density (including larger voids and higher surface area; Supplementary Table 2) than bcu. There is a modest enthalpic penalty increase for forming scu from bcu $\left(+14.6 \mathrm{~kJ} \mathrm{~mol}^{-1}\right)$ or reo $\left(+12.0 \mathrm{~kJ} \mathrm{~mol}^{-1}\right)$ as the connectivity of scu is lower (4-, 8-connected net). Because the energy of forming any of the bcu, reo and scu structures from the perfect fcu material is relatively small, the calculations hint that all these defective structures are accessible under synthesis conditions. The accessible porosities of the four observed phases have been calculated based on the identified crystal structures, and the results indicate that both surface area and pore volume increase with the framework becoming more open, following the order $\mathbf{f c u}<\mathbf{b c u}<$ reo $<$ scu (Supplementary Table 2).

Defect evolution and crystal ripening. The coexistence and comparable formation energies of missing-linker and missing-cluster defects in UiO-66 inspired us to investigate whether one could transform to the other. To this end, we synthesized UiO-66-D samples with different crystallization times. We find that the diffuse scattering peaks in PXRD become apparently weaker when the crystallization time is increased from 1 day to 3 days; concurrently, the surface area and porosity of the product decrease, as determined from the $\mathrm{N}_{2}$ sorption isotherms. In contrast, when the crystallization time is shortened to $1 \mathrm{~h}$, stronger diffuse scattering peaks and increased surface area and porosity are observed (Supplementary Fig. 12). These results indicate that the missingcluster defects gradually disappear with prolonged crystallization. Meanwhile, we note that the crystallization of UiO-66 is almost complete with a high product yield of $84 \%$ in 1 day; further extending the crystallization time to 3 days leads to little change in the product yields $(88 \%)$ but significant increase in the crystal size from $<50 \mathrm{~nm}$ to $\sim 100 \mathrm{~nm}$ (Supplementary Fig. 2). A direct conclusion from these observations is that $\mathrm{UiO}-66$ crystals undergo a dissolution-redeposition (Ostwald ripening) process that is accompanied by the evolution of structural defects. Characterization of the 3 day sample with HRTEM confirmed the fading of missingcluster domains and revealed that ordered missing-linker defects remain predominant (Supplementary Fig. 13). On the other hand, the sample collected at $1 \mathrm{~h}$ of crystallization (product yield of $48 \%$ ) has both types of defect but a higher proportion of missing clusters compared to the 1 day sample (Supplementary Figs. 12 and 13). Taken together, we discovered a simultaneous evolution of crystal size and defective structure of UiO-66. Interestingly, we found that the defect evolution in UiO-66 is strongly dependent on the amount of the modulator, formic acid; for instance, when 175 molar equivalents of formic acid relative to BDC are used in the synthesis ${ }^{25}$, the PXRD shows a reverse trend, where missingcluster defects are rapidly developed and grow in population as the crystallization time increases (Supplementary Fig. 14). The DFT assessment shows that in the absence of kinetic considerations, fcu is expected to be the thermodynamic product. However, the relatively low formation energies of the bcu, reo and scu structures suggest these structures could be accessible and, here, are in fact evidenced directly through imaging by HRTEM. The dilution of the BDC linker by modulating formic acid is critical in controlling the crystallization behaviour. At low ratios of formic acid to $\mathrm{BDC}$, the structure tends from a defective structure towards a perfect structure $(\mathbf{s c u} \rightarrow \mathbf{b c u} \rightarrow \mathbf{f c u}$ or $\mathbf{s c u} \rightarrow \mathbf{r e o} \rightarrow \mathbf{f c u}$ ), presumably driven by the exothermic changes to cohesive energy. Conversely, under high ratios of formic acid to $\mathrm{BDC}, \mathrm{UiO}-66$ grows increasing populations of missing-cluster defects (scu or reo).

Unlike ordered missing-cluster defects, which lead to additional PXRD peaks, ordered missing-linker defects are indistinguishable from the perfect structure by PXRD (Supplementary Fig. 7), but can be identified by HRTEM. In fact, we observed ordered missing-linkers in the 3-day UiO-66-D sample that would be considered nearly defect-free based on the PXRD and $\mathrm{N}_{2}$ adsorption data, as well as in UiO-66-P prepared with acetic acid as modulator (Supplementary Figs. 12 and 13). These results suggest that ordered missing-linker defects exist universally in various UiO-66 samples, including those deemed seemingly perfect.

Fine-tuning the defects and catalytic activities of UiO-66. The catalytic activity of UiO-66 has been correlated with the concentration of defects ${ }^{38,39}$. However, previous studies did not specify or distinguish the effects from missing-linker defects and missing-cluster defects $^{38-41}$. Here, the 1 day and 3 day UiO-66-D samples (hereafter denoted UiO-66-D(1d) and UiO-66-D(3d)) offer an opportunity to investigate the structure-activity relationships of this catalyst more definitively, because these samples have similar overall defect concentrations as indicated by the content of the defect-terminating formates (3.93 wt\% and $3.65 \mathrm{wt} \%$; Fig. 5a), yet at that same time different concentrations of each type of defect: UiO-66-D(1d) has substantial amounts of both types of defect, whereas UiO-66-D(3d) is deficient in missing-cluster defects and dominated by missinglinker defects. We used the model reaction of the isomerization of glucose to fructose to evaluate catalytic activities during which the defects (under-coordinated $\mathrm{Zr}^{4+}$ ) act as the catalytically active Lewis acid sites. As shown in Fig. 5b, UiO-66-D(1d) yielded markedly higher glucose conversion (47\%) than UiO-66-D(3d) (34\%) under identical reaction conditions, indicating that the catalytic sites in the configuration of missing clusters are more active than those in missing linkers. We attribute this result to the possibility that the larger cavities associated with missing-cluster defects enhance the accessibility of the active sites.

By using a post-synthesis ligand-exchange $\operatorname{method}^{37}$, we could gradually 'heal' the missing-linker defects to further tune the catalytic activity. Specifically, we prepared two samples by incubating $\mathrm{UiO}-66-\mathrm{D}(3 \mathrm{~d})$ in $\mathrm{BDC}$ solution at $80^{\circ} \mathrm{C}$ to facilitate ligand exchange 

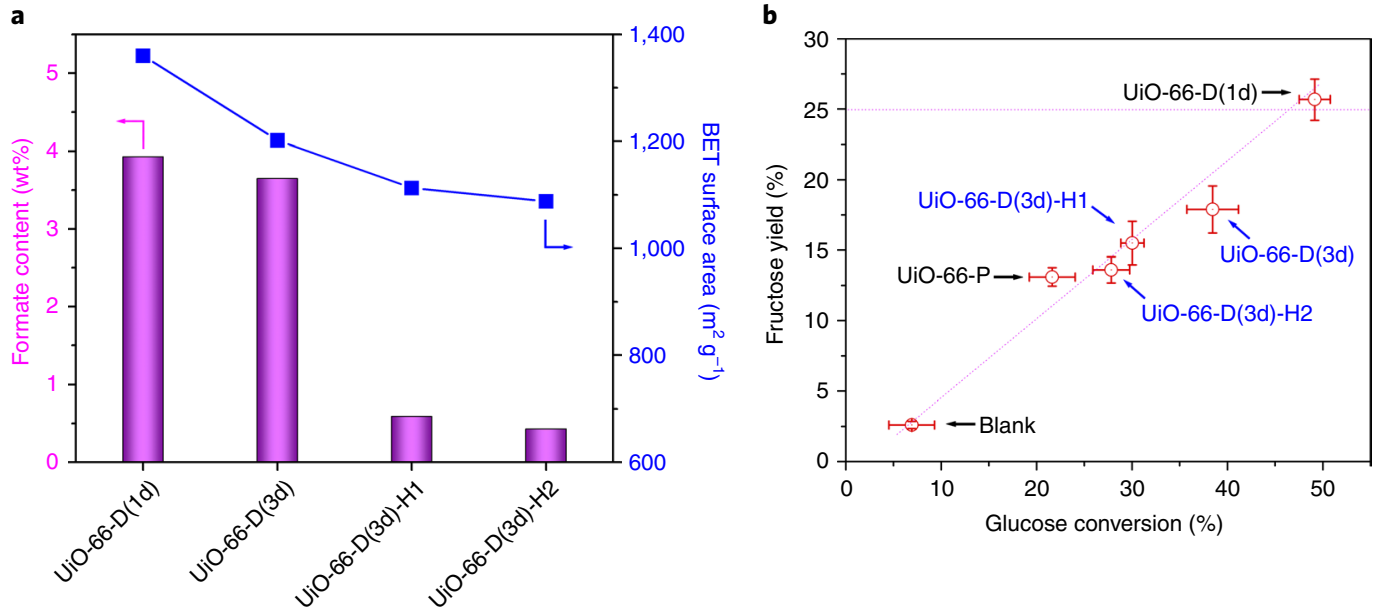

Fig. 5 | Characterization and catalytic performances of various defective UiO-66 samples. a, Formate contents determined by high-performance liquid chromatography analysis of the HF-digested solution (see Supplementary section 'Determination of the formate content in UiO-66 samples' for a detailed method) and BET surface areas derived from $\mathrm{N}_{2}$ sorption isotherms of four UiO-66 samples as labelled. $\mathbf{b}$, Catalytic performances of various UiO-66 samples in the isomerization of glucose to fructose. 'Blank' refers to the case without using a catalyst. Error bars represent standard uncertainties in the mean values calculated from five batches of repeated experiments. Dashed lines are guides to the eye.

(replacing formates with terephthalates). One sample (UiO-66$\mathrm{D}(3 \mathrm{~d})-\mathrm{H} 1)$ experienced this healing process once, while the other sample (UiO-66-D(3d)-H2) experienced it twice. The conditions of the healing process were carefully selected to ensure that ligand exchange rather than crystal dissolution and recrystallization took place, as indicated by the unaltered crystal sizes and morphologies of the healed samples (Supplementary Fig. 15). In comparison with UiO-66-D(3d), the healed samples had substantially fewer formates and smaller Brunauer-Emmett-Teller (BET) surface areas (Fig. 5a) and their structures were predominantly 'perfect' according to HRTEM (Supplementary Fig. 15), which attested to the success of the defect-healing process. When these materials were used as catalysts for glucose isomerization, their activities were in the following order: UiO-66-D(3d) > UiO-66-D(3d)-H1 > UiO-66-D(3d)-H2 (Fig. 5b), in accordance with the observed concentration of defects. These results demonstrate that the ability to tune and identify defects enables the exploration of the distinct roles of missing-linker and missing-cluster defects in the catalytic activity.

\section{Conclusion}

We directly imaged local structural defects in MOF UiO-66 by HRTEM. Studies based on diffraction techniques typically probe spatially averaged defect structures. In contrast, the use of HRTEM shows the coexistence of missing-linker and missing-cluster defects in the same crystal and their local structural ordering at the atomic scale. The high quality of the HRTEM images enables a 3D reconstruction of the missing-linker defect, in which all the structural components, including defect-terminating formate ligands, are clearly resolved. Two types of missing-cluster defect are also determined based on HRTEM. In the investigated system, missing-linker defects are found to prevail, whereas missing-cluster defects only appear in small regions of few unit cell dimensions; with prolonged crystallization, with the ripening of crystals, only missing-linker defects remain. In addition, the trend of defect evolution can be reversed by increasing the concentration of modulator. Missingcluster defects are also shown to be more catalytically active than missing-linker defects, at comparable defect concentrations. A post-synthesis ligand-exchange method was used to heal missinglinker defects, and this enabled fine-tuning of the catalytic activity for $\mathrm{UiO}-66$.
Furthermore, missing-linker defects were observed in various UiO-66 samples, including those that would have typically been assumed to be essentially defect-free, which underlines that notionally perfect materials can contain defects invisible to most characterization techniques that may influence observed variance in properties of MOFs such as gas uptake ${ }^{42,43}$. The advent of low-dose HRTEM enables the exploration of the link between the functionality of MOFs and their activity through unambiguous structure determination. We envisage that HRTEM will also be useful for the investigation of local structures in other functional materials such as covalent organic frameworks or porous cage materials ${ }^{44}$ that are sensitive to electron beams.

\section{Data availability}

The authors declare that all the data supporting the findings of this study are available within the paper and the Supplementary Information, and/or from the authors upon reasonable request.

Received: 4 July 2018; Accepted: 26 March 2019; Published online: 13 May 2019

\section{References}

1. Li, H., Eddaoudi, M., O'Keeffe, M. \& Yaghi, O. M. Design and synthesis of an exceptionally stable and highly porous metal-organic framework. Nature 402, 276-279 (1999).

2. Eddaoudi, M. et al. Systematic design of pore size and functionality in isoreticular MOFs and their application in methane storage. Science 295, 469-472 (2002).

3. Yaghi, O. M. et al. Reticular synthesis and the design of new materials. Nature 423, 705-714 (2003).

4. Horike, S., Shimomura, S. \& Kitagawa, S. Soft porous crystals. Nat. Chem. 1, 695-704 (2009).

5. Eddaoudi, M., Sava, D. F., Eubank, J. F., Adil, K. \& Guillerm, V. Zeolite-like metal-organic frameworks (ZMOFs): design, synthesis and properties. Chem. Soc. Rev. 44, 228-249 (2015).

6. Li, J.-R., Kuppler, R. J. \& Zhou, H.-C. Selective gas adsorption and separation in metal-organic frameworks. Chem. Soc. Rev. 38, 1477-1504 (2009).

7. Férey, G. et al. A chromium terephthalate-based solid with unusually large pore volumes and surface area. Science 309, 2040-2042 (2005).

8. Chui, S. S.-Y., Lo, S. M.-F., Charmant, J. P. H., Orpen, A. G. \& Williams, I. D. A chemically functionalizable nanoporous material $\left[\mathrm{Cu}_{3}(\mathrm{TMA})_{2}\left(\mathrm{H}_{2} \mathrm{O}\right)_{3}\right]_{n}$. Science 283, 1148-1150 (1999). 
9. Dissegna, S., Epp, K., Heinz, W. R., Kieslich, G. \& Fischer, R. A. Defective metal-organic frameworks. Adv. Mater. 30, 1704501 (2018).

10. Fang, Z., Bueken, B., De Vos, D. E. \& Fischer, R. A. Defect-engineered metal-organic frameworks. Angew. Chem. Int. Ed. 54, 7234-7254 (2015).

11. Bennett, T. D., Cheetham, A. K., Fuchs, A. H. \& Coudert, F.-X. Interplay between defects, disorder and flexibility in metal-organic frameworks. Nat. Chem. 9, 11-16 (2017).

12. Lee, J. et al. Metal-organic framework materials as catalysts. Chem. Soc. Rev. 38, 1450-1459 (2009).

13. Sumida, K. et al. Carbon dioxide capture in metal-organic frameworks. Chem. Rev. 112, 724-781 (2012).

14. Shoaee, M., Agger, J. R., Anderson, M. W. \& Attfield, M. P. Crystal form, defects and growth of the metal organic framework HKUST-1 revealed by atomic force microscopy. CrystEngComm 10, 646-648 (2008).

15. Ling, S. \& Slater, B. Dynamic acidity in defective UiO-66. Chem. Sci. 7, 4706-4712 (2016)

16. Cavka, J. H. et al. A new zirconium inorganic building brick forming metal-organic frameworks with exceptional stability. J. Am. Chem. Soc. 130, 13850-13851 (2008)

17. Jakobsen, S. et al. Structural determination of a highly stable metal-organic framework with possible application to interim radioactive waste scavenging: Hf-UiO-66. Phys. Rev. B 86, 125429 (2012).

18. Falaise, C. et al. Three-dimensional MOF-type architectures with tetravalent uranium hexanuclear motifs $\left(\mathrm{U}_{6} \mathrm{O}_{8}\right)$. Chem. Eur. J. 19, 5324-5331 (2013).

19. Shearer, G. C. et al. Defect engineering: tuning the porosity and composition of the metal-organic framework UiO-66 via modulated mynthesis. Chem. Mater. 28, 3749-3761 (2016).

20. Shearer, G. C. et al. Tuned to perfection: ironing out the defects in metal-organic framework UiO-66. Chem. Mater. 26, 4068-4071 (2014).

21. Valenzano, L. et al. Disclosing the complex structure of UiO-66 metalorganic framework: a synergic combination of experiment and theory. Chem. Mater. 23, 1700-1718 (2011).

22. $\mathrm{Wu}, \mathrm{H}$. et al. Unusual and highly tunable missing-linker defects in zirconium metal-organic framework UiO-66 and their important effects on gas adsorption. J. Am. Chem. Soc. 135, 10525-10532 (2013).

23. Øien, S. et al. Detailed structure analysis of atomic positions and defects in zirconium metal-organic frameworks. Cryst. Growth Des. 14, 5370-5372 (2014).

24. Trickett, C. A. et al. Definitive molecular level characterization of defects in UiO-66 crystals. Angew. Chem. Int. Ed. 54, 11162-11167 (2015).

25. Cliffe, M. J. et al. Correlated defect nanoregions in a metal-organic framework. Nat. Commun. 5, 4176 (2014).

26. Schrimpf, W. et al. Chemical diversity in a metal-organic framework revealed by fluorescence lifetime imaging. Nat. Commun. 9, 1647 (2018).

27. Hashimoto, A., Suenaga, K., Gloter, A., Urita, K. \& Iijima, S. Direct evidence for atomic defects in graphene layers. Nature 430, 870-873 (2004).

28. Platero-Prats, A. E. et al. Bridging zirconia nodes within a metal-organic framework via catalytic Ni-hydroxo clusters to form heterobimetallic nanowires. J. Am. Chem. Soc. 139, 10410-10418 (2017).

29. Mehdi, B. L. et al. Low-dose and in-painting methods for (near) atomic resolution STEM imaging of metal organic frameworks (MOFs). Microsc. Microanal. 23, 1804-1805 (2017).

30. Mayoral, A., Sanchez-Sanchez, M., Alfayate, A., Perez-Pariente, J. \& Diaz, I. Atomic observations of microporous materials highly unstable under the electron beam: the cases of Ti-doped $\mathrm{AlPO}_{4}-5$ and $\mathrm{Zn}-\mathrm{MOF}-74$ ChemCatChem 7, 3719-3724 (2015).

31. Leus, K. et al. Atomic layer deposition of Pt nanoparticles within the cages of MIL-101: a mild and recyclable hydrogenation catalyst. Nanomaterials 6, 45 (2016).

32. Zhu, Y. et al. Unravelling surface and interfacial structures of a metal-organic framework by transmission electron microscopy. Nat. Mater. 16, 532-537 (2017).

33. Zhang, D. et al. Atomic-resolution transmission electron microscopy of electron beam-sensitive crystalline materials. Science 359, 675-679 (2018).

34. Sakamoto, Y. et al. Direct imaging of the pores and cages of threedimensional mesoporous materials. Nature 408, 449-453 (2000).
35. Han, Y. et al. A tri-continuous mesoporous material with a silica pore wall following a hexagonal minimal surface. Nat. Chem. 1, 123-127 (2009).

36. Vandichel, M. et al. Active site engineering in UiO-66 type metal-organic frameworks by intentional creation of defects: a theoretical rationalization. CrystEngComm 17, 395-406 (2015).

37. Taddei, M., Wakeham, R. J., Koutsianos, A., Andreoli, E. \& Barron, A. R. Post-synthetic ligand exchange in zirconium-based metal-organic frameworks: beware of the defects! Angew. Chem. Int. Ed. 57, 11706-11710 (2018).

38. Vermoortele, F. et al. Synthesis modulation as a tool to increase the catalytic activity of metal-organic frameworks: the unique case of UiO-66(Zr). J. Am. Chem. Soc. 135, 11465-11468 (2013).

39. Korzyński, M. D., Consoli, D. F., Zhang, S., Román-Leshkov, Y. \& Dincă, M. Activation of methyltrioxorhenium for olefin metathesis in a zirconium-based metal-organic framework. J. Am. Chem. Soc. 140, 6956-6960 (2018).

40. Liu, Y., Klet, R. C., Hupp, J. T. \& Farha, O. Probing the correlations between the defects in metal-organic frameworks and their catalytic activity by an epoxide ring-opening reaction. Chem. Commun. 52, 7806-7809 (2016).

41. De Mello, M. D. \& Tsapatsis, M. Selective glucose-to-fructose isomerization over modified zirconium UiO-66 in alcohol media. ChemCatChem 10, 2417-2423 (2018).

42. Sholl, D. S. \& Lively, R. P. Defects in metal-organic frameworks: challenge or opportunity? J. Phys. Chem. Lett. 6, 3437-3444 (2015).

43. Park, J., Howe, J. D. \& Sholl, D. S. How reproducible are isotherm measurements in metal-organic frameworks? Chem. Mater. 29, 10487-10495 (2017)

44. Jones, J. T. A. et al. Modular and predictable assembly of porous organic molecular crystals. Nature 474, 367-371 (2011).

\section{Acknowledgements}

This research was supported by Competitive Center Funds (FCC/1/1972-19) to Y.H. and M.E. from King Abdullah University of Science and Technology. This research used resources of the Core Labs of King Abdullah University of Science and Technology. Yi.Z. acknowledges financial support from the National Natural Science Foundation of China (21771161) and the Thousand Talents Program for Distinguished Young Scholars. S.L. and B.S. are thankful to the Materials Chemistry Consortium (EPSRC: EP/L000202) for provision of computer time on ARCHER UK National Supercomputing Service. B.S. acknowledges the Royal Society for financial support through an industry fellowship (F160062). The authors acknowledge helpful discussions with A. Goodwin, M. Cliffe and G. Shearer.

\section{Author contributions}

Y.H., D.Z. and M.E. conceived and designed the experiments. L.L. acquired the low-dose high-resolution HRTEM images. Z.C., J.W., Y.B. and K.A. designed and synthesized MOF samples. Z.C., J.W. and Y.B. performed X-ray diffraction and $\mathrm{N}_{2}$ adsorption characterizations. L.L., D.Z., Yi.Z. and Y.H. processed and analysed the HRTEM images. D.Z. solved the 3D structure of the missing-linker defect. S.L. and B.S. performed theoretical calculations. J.W., Yu.Z. and K.-W.H. designed and performed the catalytic reactions. Y.H., D.Z., M.E., S.L. and B.S. wrote the manuscript and all authors commented on the manuscript.

\section{Competing interests}

The authors declare no competing interests.

\section{Additional information}

Supplementary information is available for this paper at https://doi.org/10.1038/ s41557-019-0263-4.

Reprints and permissions information is available at www.nature.com/reprints. Correspondence and requests for materials should be addressed to D.Z., B.S., M.E. or Y.H.

Publisher's note: Springer Nature remains neutral with regard to jurisdictional claims in published maps and institutional affiliations.

(C) The Author(s), under exclusive licence to Springer Nature Limited 2019 